\title{
THE MECHANISM OF PELVIC SUPPORT
}

\author{
G. B. Gibson, M.A.O., F.R.C.S.(Edin.), M.R.C.O.G. \\ Gynacologist, N. Armagh Hospital Group, N. Ireland
}

A KNOWLEDGE of surgical anatomy is the first pre-requisite in the evolution of sound surgical practice. The repair of genital prolapse is the most frequent operation in gynæcology with the exception of dilatation and curettage, and yet it is a curious fact that details of pelvic anatomy and the mechanism of pelvic support are still open to argument among gynæcologists.

Bonney (1936) likened the vagina to the inturned finger of a rubber glove and the forces it withstood to the compression of air within the glove. Thickening of the rubber, narrowing at the point of inversion and the attachment of elastic bands to the inverted finger made eversion more difficult. Similarly, a thick muscular vaginal wall, the sphincteric action of the pelvic diaphragm, and attachment of the endo-pelvic ligaments to the cervix prevented eversion of the vagina. As a general concept this is fine, although Mengert (1936) and Berglas and Rubin (1953) would disagree, for different reasons.

The former experimented upon the fresh cadaver, applying traction to the cervix while dividing each of the structures which could support it. He concluded that the lateral endo-pelvic ligament was all important and that muscular support was negligible. The rarity of genital prolapse in association with a complete perineal tear is often quoted in support of this view though associated scarring of the cervix and parametrium may explain the absence of uterine descent. The latter workers did extensive histological investigation, using massive sections of the whole pelvis. They were unable to find any evidence of thickening of the endo-pelvic areolar tissue and concluded that the endo-pelvic ligaments were a myth. In their view support is entirely muscular and they designate the essential muscles as the levator crura (the pubo-coccygeus muscles) and the levator plate (the perineal decussation of the two muscles).

The writer's conception of pelvic anatomy has changed with increasing experience and, as students still seem to start where he began, it may be of value to describe his changing views.

\section{First Impressions of Pelvic Anatomy}

The textbook description of pelvic anatomy, illustrated by three standard diagrams (Figs. I, 2 and 3) has shown little modification over the last half-century. This description suggests that the vagina follows the line of the pelvic axis, having a gentle curve, concave forwards. Figs. I and 2 suggest that it rises well above the plane of the pelvic diaphragm and is supported at its upper end by the cervix. The cervix is fixed in the midposition of the pelvis by three sets of ligaments, pubo-cervical, lateral and utero-sacral, arranged in pairs (Fig. 3). Smout and Jakoby (1948) liken this arrangement to a cartwheel, the cervix representing the hub, the ligaments the spokes and the bony pelvis the rim of the wheel.

This general idea of pelvic anatomy led the

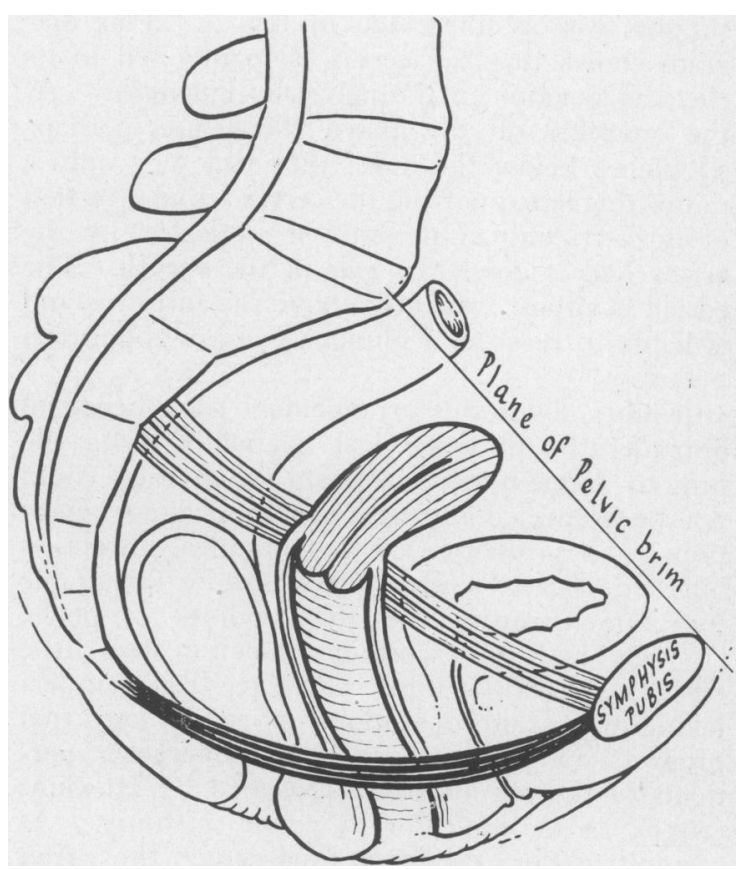

FIG. I-Lateral view. 


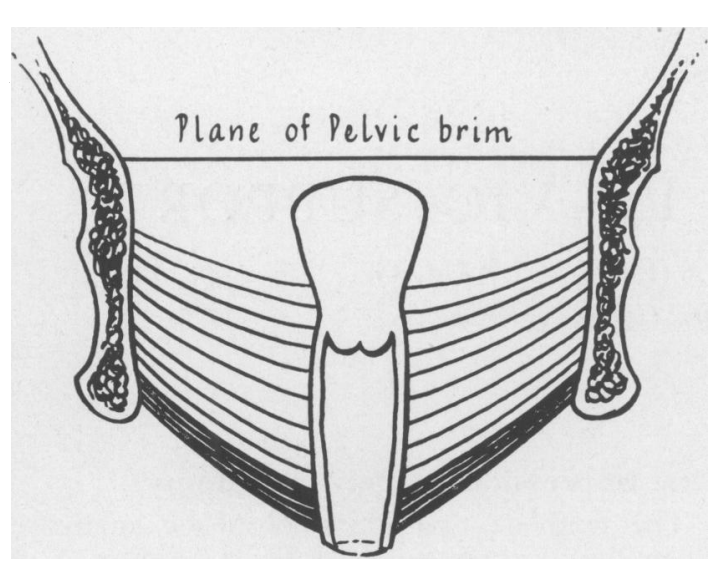

Fig. 2-Antero-posterior view.

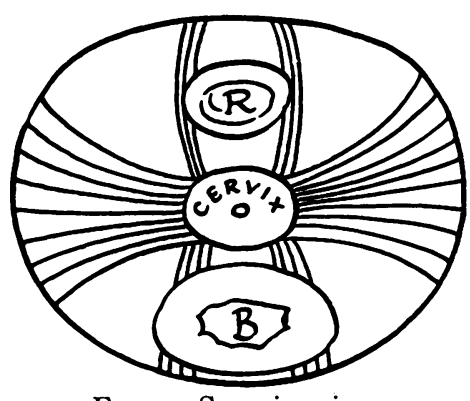

Fig. 3-Superior view.

writer to believe that the mechanism of pelvic support was as illustrated in Fig. 4. This diagram shows that the cervix is maintained in its elevated position in the pelvis by ligaments. As the muscles of the pelvic floor are perhaps $2 \frac{1}{2}$ inches below this level they can play only a minor role in supporting the cervix. The direction of intra-abdominal pressure is indicated by the arrow and is down the axis of the vagina. The cervix is situated directly above the introitus and is liable to react as a plunger to intra-abdominal pressure.

Doubts about this arrangement soon began to obtrude, the pubo-cervical ligaments being the first to come under suspicion when they could not be identified during repair and sling operations. These doubts led to anatomical dissection but a dessicated corpse proved quite unsuitable for demonstrating the finer points of pelvic anatomy and post-mortem dissection was littlc better. It was not until the writer began undertaking the major operations in gynæcology that progress was made. A Wertheim operation, particularly the modification described by Howkins $(195 \mathrm{I})$, is the educator in pelvic anatomy. As a result of the experience thus gained the writer reached the following conclusions:

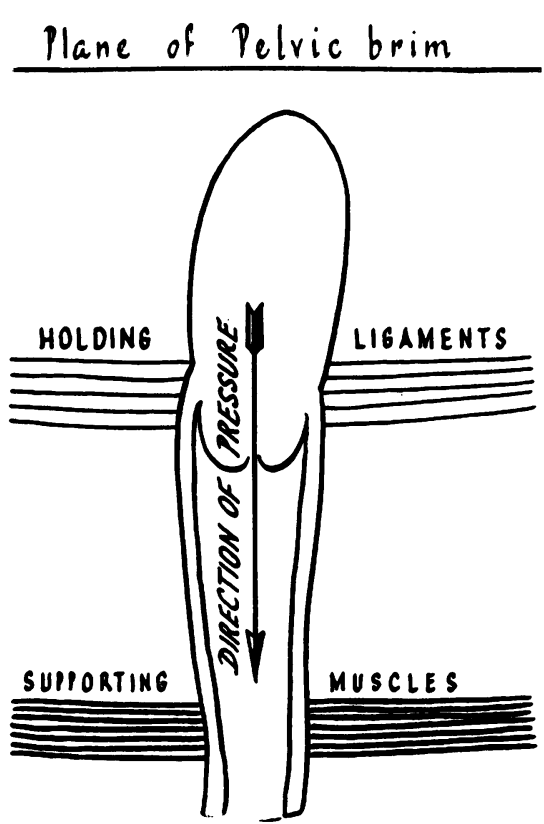

Fig. 4-Mechanics of support.

I. The lateral ligament is a definite structoir $\vec{B}$ and not a leash of blood vessels, surrounded $\$$ \% loose areolar tissue, as suggested by Berglas ầं Rubin (1953).

2. This ligament is attached to the cervix an to the full extent of the lateral vaginal wall.

3. There are no other endo-pelvic ligaments though the posterior edge of the lateral ligamen? could be designated as the utero-sacral ligamente However, until the uterus is pulled upwards, putting this portion of the lateral ligament on the stretch, it is in direct continuity with the remainder of the ligament. Having reached this conclusion the writer found that Fothergill (I92I) stated:- Clinical experience gradually taught me that the uterus, vagina and bladder are mainly kept i. their places by the lateral combination of unว striped muscle and connective tissue known as the parametrium and paracolpos. Finding that several anatomists had come to the same cono clusion, I brought their names and work before the Royal Society of Medicine in 1907. Tho facts then mentioned have been rediscovered with surprising frequency in various parts of the्ట world'.

4. The cervix is situated as close to the sacrum as the rectum will permit and is certainly not tethered in the mid-point of the pelvis.

5. The course of the vagina follows the floor of the pelvis and the vagina rests on the perineat structures. 


\section{Revised Impressions of Pelvic Anatomy}

These conclusions indicated that the mechanism of pelvic support differed from that outlined earlier and to confirm this a radiological investigation was undertaken. The rectum, vagina, uterus and bladder were filled with radio opaque fluids, either alone or severally. Three views were taken in each case, (a) erect lateral, (b) erect antero-posterior, and (c) supero-inferior brim view. Approximately 30 views were obtained. Figs. 5 and 6 are line drawings from lateral views, the patients being a nullipara and a multipara with no evidence of prolapse. Fig. 5 shows that the vagina is not curved, with the concavity forwards. It bends backwards sharply at the junction of the lower one-third and the upper two-thirds. In Fig. 6 this angle has disappeared, presumably due to lack of tone in the perineal body, associated with multiparity. In both figures the vagina lies

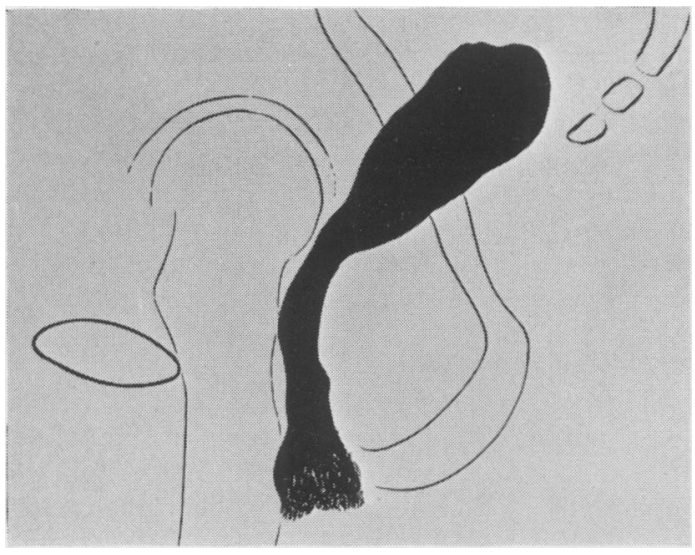

FIG. 5-Colpograph-lateral view. Nullipara.

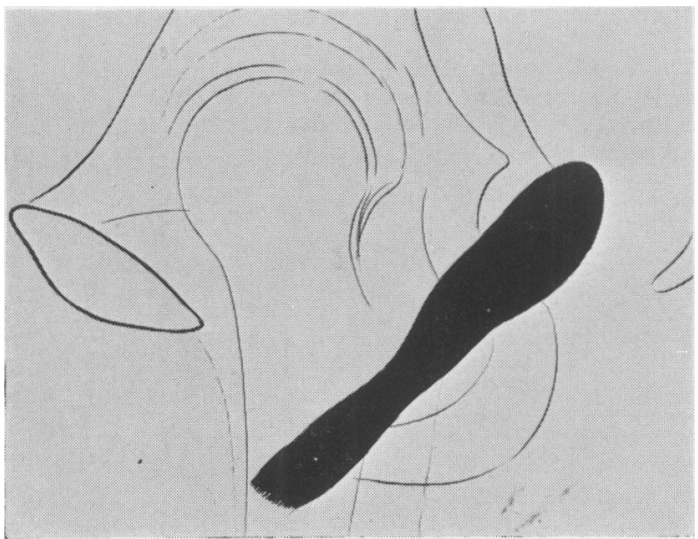

FIG. 6-Colpograph-lateral view. Multipara. on the upper surface of the levatores ani, rectum and coccyx.

The radiological investigation revealed the major difficulty in preparation of diagrams to illustrate pelvic anatomy, namely, the distortion produced by a $60^{\circ}$ tilt of the pelvis. To get over this difficulty a model was prepared and this was photographed. In Fig. 7, a lateral view of the model, the course of the vagina is shown. It lies on the lower layer of structures, the levatores ani, coccyx and sacrum, with the rectum intervening between them. Attached to the lateral wall of the vagina is the cut edge of the lateral ligament. The posterior end of this ligament is continued

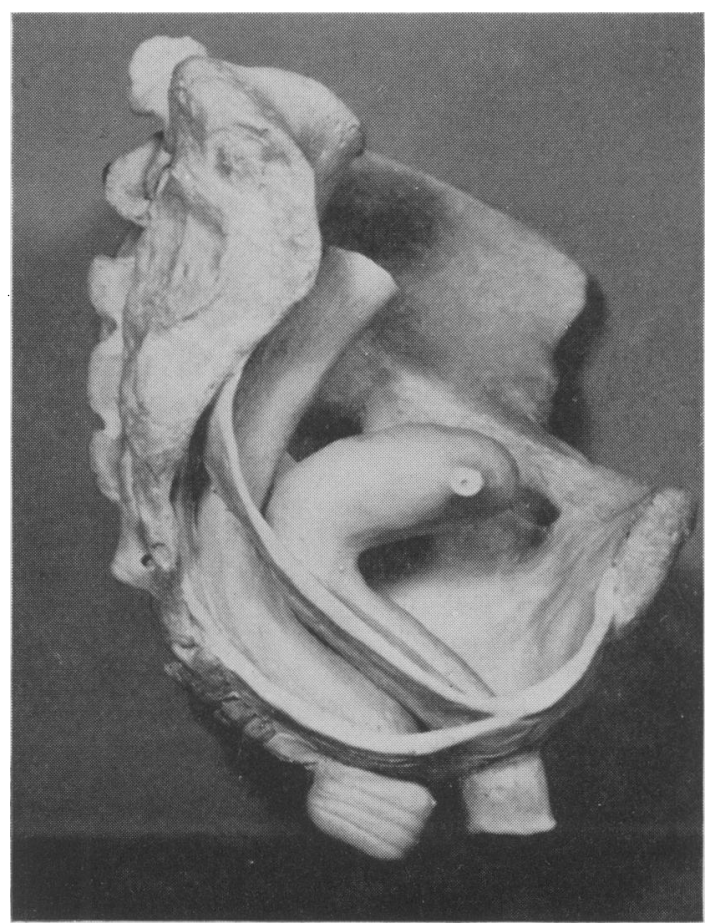

FIG. 7-Lateral view of model.

backwards to the sacrum and may be described as the utero-sacral ligament. Fig. 8 is a superior view of the model in which the fan-shaped lateral ligament is shown lying in contact with the superior surface of the pelvic diaphragm and supported by it.

A study of this model suggests that the mechanism of pelvic support is as illustrated in Fig. 9. An arrow represents the direction of intraabdominal pressure. This is at right angles to the vaginal axis and tends to flatten the vagina against the muscle cradle below it, a mechanism similar 


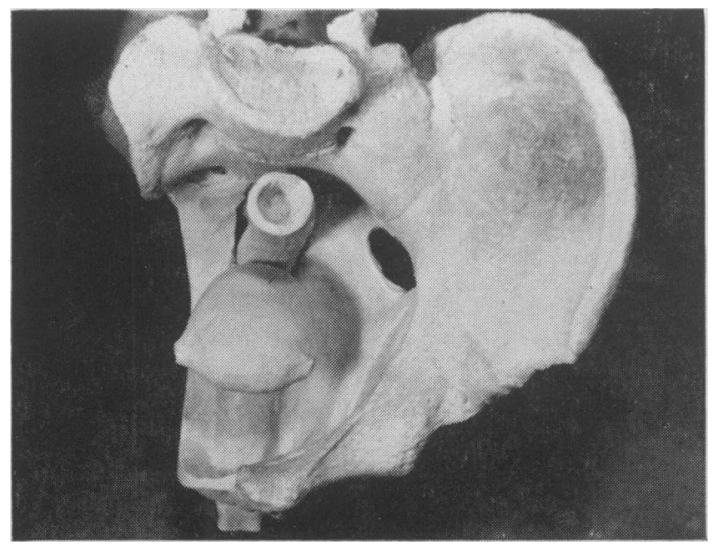

FIG. 8-Superior view of model.

to that aimed at in a Coffey ureteric transplant. The lateral ligaments and pelvic diaphragm are parallel to each other and complementary in rendering support to the genital tract. As the muscle hiatus opens due to perineal damage, the ligamentary support of the anterior vaginal wall carries an excessive load and a cystocele develops. Damage to the posterior segment of the lateral ligaments permits the cervix to shift forwards in the pelvis and when it is in line with the introitus uterine prolapse occurs.

If this view of the mechanism of pelvic support is accepted it follows that the aims of a repair operation should be: (a) The plication of the superior fibres of the lateral ligament between the bladder and vagina; $(b)$ backward displacement of the cervix in the pelvis by means of Fothergill stitches; $(c)$ the plication of the inferior fibres of

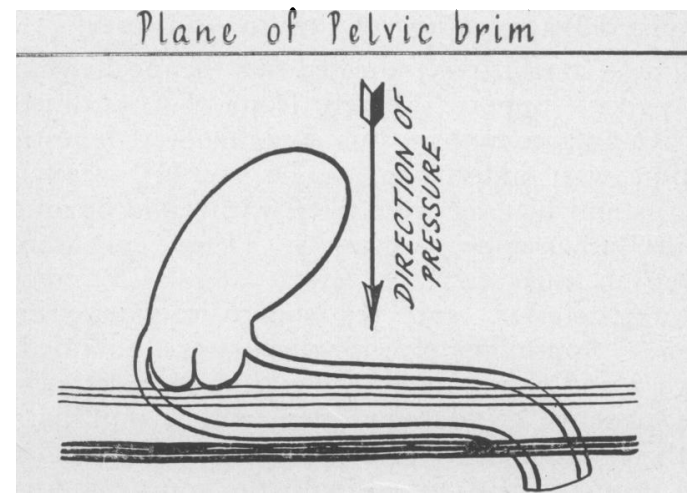

FIG. 9-Mechanics of support.

the lateral ligament between the rectum an vagina; and $(d)$ reconstruction of the perineas body.

\section{Summary}

The mechanism of pelvic support has been discussed. Clinical and radiological investigation suggest that the course of the vagina in the pelvis differs from the standard description in textbookson Thus intra-abdominal pressure is applied at right. angles to the vagina and not along its axis. The pressure tends to flatten the vagina against $\overrightarrow{0}+\overrightarrow{\mathrm{s}}$ supporting structures rather than to evert it.

\section{Acknowledgments}

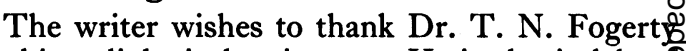
for his radiological assistance. $\mathrm{He}$ is also indebte to Mr. G. A. Smith, Medical Artist, for thet preparation of the model and for his drawings.

\section{REFERENCES}

Berglas, B., and Rubin, I. C. (1953): Study of Supportive Structures of the Uterus, Surg. Gynec. Obstet., 97, 277. Bonney, V. (1934): The Principles that should Underline all Operations for Prolapse, F. Obstet. Gynac. Brit. Emp. 4I, 669.

Fothergill, W. E. (1921): The End Results of Vaginal Operation for Genital Prolapse, Ibid., 28, 251 .

H owkins, J. (195 I): Synchronous Combined Abdomino-vaginal Hysterectomy, Lancet, i, 872.

M engfrt, W. F. (1936): Mechanics of Uterine Support and Position, Amer. . Obstet. Gynec., 31, 775.

SM оuт, C. F. V., and JАкову, F. (1948): Gynæcological and Obstetrical Anatomy, p. 66, 2nd Edition. London: Edwarc Arnold \& Co. 